\title{
A panel analysis on preventing and controlling efficiency of COVID-19 pandemic
}

\section{Ming Guan ( $\nabla$ gming0604@163.com )}

\section{Research}

Keywords: dead cases, confirmed cases, healed cases, recovered cases, COVID-19

Posted Date: May 6th, 2020

DOI: https://doi.org/10.21203/rs.3.rs-26006/v1

License: (c) (1) This work is licensed under a Creative Commons Attribution 4.0 International License. Read Full License 
Title Page

Title: A panel analysis on preventing and controlling efficiency of COVID-19 pandemic

Author's name: Ming Guan, Dr, Prof.

Author's affiliation:

1. International Issues Center, Xuchang University, Xuchang, Henan, China

2. Family Issues Center, Xuchang University, Xuchang, Henan, China

3. School of Business, Xuchang University, Xuchang, Henan, China

Author's email address: gming0604@163.com

Telephone number: $\quad 86+13409360114$

Postal address: Road Bayi 88,Xuchang, China 


\section{A panel analysis on preventing and controlling efficiency of COVID-19 pandemic}

BACKGROUND: Currently, the globe is making efforts to curb the spreading of coronavirus disease 2019 (COVID-19). However, only a few studies have examined the associations among spreading growth, treatment efficiency, and death increase. This study aimed to examine the associations of COVID-2019 dead cases, healed cases, and recovered cases.

METHODS: Data from China Data Lab in Harvard Dataverse with the China (January 22, 2020 to March 18, 2020), United States of America (USA, March 17, 2020 to April 12, 2020), and the world (January 22, 2020 to April 4, 2020) were analyzed. The main variables included in the analysis were the numbers of COVID-2019 total confirmed cases (TC), total dead cases (TD), newly dead cases (ND), newly confirmed cases (NC), newly healed cases (NH), total healed cases (TH), and newly recovered cases (NR). Pooled, dynamic, and event study analyses were conducted to reflect the associations among them.

RESULTS: Descriptive analyses showed that NH/NC ratio in China are bigger than $\mathrm{NR} / \mathrm{NC}$ in USA and the world. Pooled analysis showed that various roles of regions in $\mathrm{NH}$ and NR in a specific sample. Dynamic analysis showed significant roles of lags and NC in NH and NR. Panel event study showed that key events influence ND and NH in China significantly and NR in the world rather than NR in USA. 
CONCLUSION: The findings in this study indirectly confirmed the relationship between spreading growth, treatment efficiency, and death increase. China's control strategies of COVID-19 pandemic are worth of learning by the globe.

KEYWORD: dead cases; confirmed cases; healed cases; recovered cases; COVID-19;

\section{Introduction}

Now, health agencies worldwide are facing the challenges of the coronavirus disease 2019 (COVID-19) endemic. Saliva droplets and direct and indirect contact via surfaces identified as the possible risk factors for interhuman transmission of COVID-19 [1]. Since late January, massive public health interventions have been implemented by national bodies to contain the spread of the virus and infection. Without validated drugs [2], successful strategies for COVID-19 from China such as wartime control measures [3], active surveillance, contact tracing, quarantine, and social distancing [4], enhanced traffic control bundling [5], and outbreak city shutdown [6]result in dramatic reductions in number of daily newly confirmed cases and deaths. Under the condition of inter-human transmission and lockdown measures, the temporal dynamics and characteristics of the COVID-19 epidemic in a high-risk city were reported in China [7]. In order to reduce morbidity and mortality, several medical decision-making tools have the potential to be a major breakthrough in efforts to control the current pandemic [8-10].

Despite travel restrictions and border control measures [11] and travel limitations [12], transmission law of COVID-19 has rapidly spread across the globe. Several 
theoretical studies depicted the COVID-19 increase of transmission between persons [13]. Based on the publicly available epidemiological data, a simulation forecast a trend of the COVID-19 spreading in Hubei, China [14]. With estimated 2.68 reproductive number for COVID-19, a study inferred that COVID-19 epidemics are already growing exponentially in multiple major cities of China with a lag time behind the Wuhan outbreak of about 1-2 weeks [15]. A simulation study indicated that around 4,000 and 18,300 people will die of COVID-19 in China and across the world, respectively [16]. The global spread of COVID-19 pandemic accelerates the speed and volume of clinical trials launched to investigate potential therapies for COVID-19 [17].

From December 12, 2019 till now, a series of daily policies and regulations released by the Chinese government, global organizations, and western countries which could be documented in China Data Lab[18]. A growing number of countries impose a nationwide containment policy in the fight against epidemic COVID-19 viral infection. A series of extreme measures in mitigating against further development of contagion were released for the national public health interest. Thus, in order to indirectly assess national struggling efforts against COVID-19 pandemic, this study with daily cases aims to analyze the associations of ND, TD, NH, TH, and NR with NC, TC, ND, and TD.

\section{Methods}

\section{Data}


China COVID-19 daily cases included the numbers of COVID-2019 TC, TD, $\mathrm{ND}, \mathrm{NC}, \mathrm{NH}$, and $\mathrm{TH}$ in China province-level units and city-level units from January 22, 2020 to March 18, 2020 [19]. United States of America (USA) COVID-19 daily cases included the numbers of COVID-2019 ND, NC, and newly recovered cases (NR) with state and county-level data available from March 17, 2020 to April 12, 2020 [20]. The World (outside Antarctica, China, and USA) COVID-19 daily cases included the numbers of COVID-2019 ND, NC, and NR from January 22, 2020 to April 4, 2020 [21]. China dataset contains information on 31 provinces and 181 cities. The USA dataset contains information on 51 states and 3143 counties. World dataset contains information on 246 countries and regions.

\section{Pooled regression analysis}

Here, OLS linear regression is employed to estimate:

$\mathrm{y}_{\mathrm{st}}=\alpha+\mathrm{NC}_{\mathrm{st}}+$ Sregion $_{\mathrm{st}}+\mu_{\mathrm{s}}+\lambda_{\mathrm{t}}+\mathrm{u}_{\mathrm{st}}$

where $y_{\text {st }}$ is an outcome of interest for province/city s and time t. Thus, the estimated model is of the general form in China province-level units:

$\mathrm{ND}_{\mathrm{st}}=\alpha+\mathrm{NC}_{\mathrm{st}}+$ region $_{\mathrm{st}}+\mu_{\mathrm{s}}+\lambda_{\mathrm{t}}+\mathrm{u}_{\mathrm{st}}$

$\mathrm{TD}_{\mathrm{st}}=\alpha+\mathrm{TC}_{\mathrm{st}}+$ region $_{\mathrm{st}}+\mu_{\mathrm{s}}+\lambda_{\mathrm{t}}+\mathrm{u}_{\mathrm{st}}$

The estimated model is of the general form in China city-level units:

$$
\begin{aligned}
& \mathrm{NH}_{\mathrm{st}}=\alpha+\mathrm{NC}_{\mathrm{st}}+\mathrm{ND}_{\mathrm{st}}+\text {-region }_{\mathrm{st}}+\mu_{\mathrm{s}}+\lambda_{\mathrm{t}}+\mathrm{u}_{\mathrm{st}} \\
& \mathrm{TH}_{\mathrm{st}}=\alpha+\mathrm{TC}_{\mathrm{st}}+\mathrm{TD}_{\mathrm{st}}+\text { rregion }_{\mathrm{st}}+\mu_{\mathrm{s}}+\lambda_{\mathrm{t}}+\mathrm{u}_{\mathrm{st}}
\end{aligned}
$$

The estimated model of the general form in the USA with states, USA with counties and World with countries: 
$\mathrm{NR}_{\mathrm{st}}=\alpha+\mathrm{NC}_{\mathrm{st}}+\mathrm{ND}_{\mathrm{st}}+$ Eregion $_{\mathrm{st}}+\mu_{\mathrm{s}}+\lambda_{\mathrm{t}}+\mathrm{u}_{\mathrm{st}}$

\section{Dynamic estimation}

Here, a dynamic panel corresponding to a series of lag coefficients and covariates is employed to analyze the associations of $\mathrm{ND}, \mathrm{TD}, \mathrm{NH}, \mathrm{TH}$, and $\mathrm{NR}$ with their lags and covariates. Arellano and Bond estimator (AB estimator) and Arellano and Bover/Blundell and Bond system estimator (ABS estimator) are employed to explore the associations.

\section{Panel event study}

Here, a panel event study corresponding to a difference-in-difference style model with a series of lag and lead coefficients and confidence intervals with the method implemented by the program EVENTDD in Stata [22] is employed to analyze how the key events influence ND, TD, NH, TH, and NR, respectively. In the context, there three key events were adopted as treatments in China province-level units and city-level units, USA state-level units and county-level units, and The World (outside Antarctica, China, and USA), respectively. Here, three key events were selected to carry out event study with difference-in-difference. On February 5, 2020, China releases tax exemption \& loan policies to beef up coronavirus containment (http://en.nhc.gov.cn/2020-02/06/c_76511.htm). Coronavirus Guidelines for America is issued on March 16, 2020 in USA (https://www.whitehouse.gov/briefings-statements/coronavirus-guidelines-america/).

On March 11, 2020, WHO characterizes COVID-19 as a pandemic (https://www.who.int/emergencies/diseases/novel-coronavirus-2019/events-as-they-ha 
ppen).

All analyses were performed with Stata (Version 15, Stata Corporation, College Station, Texas).

\section{Results}

\section{Descriptive analyses}

Table 1 shows the descriptive statistics for number of confirmed, dead, healed, and recovered overall COVID-19 cases. Overall, there were 1984 observations, 21440 observations, 254583 counties observations, 4233 observations, and 18,426 observations of COVID-19 cases included in this analysis during 64-day China province-level units study, 64-day China city-level units study, 83-day USA states study, 64-day USA counties study, and 74-day world study. Thus, the mean values of NC/ND, TC/TD, NC/NH, ND/NH, TC/TH, TD/TH, NC/NR, and ND/NR could reflect the treatment efficiency of COVID-19 pandemic. The mean NC/ND ratios in China province-level units and city-level units are 25.01 and 24.67, respectively, while mean NC/ND ratios in USA state-level units, USA county-level units, and the world are 59.52, 220.88, and 18.00, respectively. The mean TC/TD ratio in China is 30.32. Regarding $\mathrm{NC} / \mathrm{NH}$, the mean ratio in China city-level units is 1.16 , while mean NC/NR ratios in USA states, USA counties, and the world are $1531.19,4591.14$, and 6.817, respectively. Regarding NH/ND, the mean ratio in China city-level units is 21.20, while mean ND/NR ratios in USA states, USA counties, and the world are $25.73,20.79$, and 0.38 , respectively. The mean ratios of TC/TH and TH/TD are 2.23 and 13.45 in China city-level units, respectively. 
[Table 1]

\section{Pooled analysis}

In table 2, several important findings were obtained. In China, NC had significantly positive association with ND and negative association with $\mathrm{NH}$. TC had significantly positive association with TD and TH. Central China, East China, and Western China had significantly negative association with ND, while Northeast China and Hubei province had significantly positive association with ND. Central China, East China, Northeast China, Western China, and Hubei province had significantly negative association with TD and had significantly positive association with $\mathrm{NH}$. Northeast had significantly negative association with TH, while Western, and Hubei province had significantly positive association with TH. In USA, ND, Mid-West Region, New England, Southwest Region, and West Region had significantly positive association with NR. In the World, NC, Asia, and Middle East had significantly positive association with NR. Similarly, NC had significantly positive association with ND in China and NR in the world. NC had significantly negative association with NH in China. ND had significantly positive association with NR in USA. TC had significantly positive association with TD and TH in China.

[Table 2] 


\section{Dynamic analysis}

The dynamic panel regression results between confirmed cases and dead cases in China province-level units are presented in Table 3. In AB estimator, lags (23-42, 47, 49, and 51-55) values of ND had significantly positive association with ND. Lags (6-9, 12-22, and 44-45) values of ND had significantly negative association with ND. In ABS estimator, lags (24-42 and 48-55) values of ND had significantly positive association with using ND. Lags (6-9, 12-23, and 44-45) values of ND had significantly negative association with ND. In AB estimator, lags (19, 24, 52, and 54) values of TD had significantly positive association with TD. Lags (13, 25, and 50) values of ND had significantly negative association with ND. In ABS estimator, lags (10, 17, and 24) values of TD had significantly positive association with TD. Lags (13, 30, and 43) values of TD had significantly negative association with TD. Similarly, NC had significantly negative association with ND in China province-level units.

[Table 3]

The dynamic panel regression results between confirmed cases, dead cases and healed cases in China city-level units are presented in Table 4. In AB estimator, lags (1-59) values of NH had significantly positive association with NH. Lags (60) values of $\mathrm{NH}$ had significantly negative association with $\mathrm{NH}$. In ABS estimator, lags (5-8, $10-12,15-22,24-26,28,32,39-42$, and 44) values of $\mathrm{NH}$ had significantly positive 
association with using NH. Lags (1-3, 29-30, 34-36, and 60) values of NH had significantly negative association with $\mathrm{NH}$. In AB estimator, lags (1-5, 11, 15, 24, 28, $32,37,39-41,44,52$, and 56) values of $\mathrm{TH}$ had significantly positive association with TH. Lags (7, 9, 12, 16, 23, 26, 27, 29, 34-35, 42, 45, and 51) values of NH had significantly negative association with NH. In ABS estimator, lags (1-5, 11, 15, 24, 28, $32,37,39-41,44,52$, and 56) values of TH had significantly positive association with TH. Lags $(7,9,12,16,23,26,27,29,34-35,42,45$, and 51) values of TH had significantly negative association with TH. Similarly, ND had significantly positive associations with $\mathrm{NH}$ in China city-level units. TD had significantly positive associations with TH in China city-level units.

[Table 4]

The dynamic panel regression results between NC, ND, and NR in USA and the world are presented in Table 5. In AB estimator, lags (54) values of NR had significantly positive association with NR. Lags (2, 4, 6, 8, 10, and 12) values of NR had significantly negative association with NR. In ABS estimator, lags $(1,3,5,7,9$, and 11) values of NR had significantly positive association with using NR. Lags (2, 4, $6,8,10$, and 12) values of NR had significantly negative association with NR. In AB estimator, lags $(2,4,6,22,36,52$, and 53$)$ values of TR had significantly positive association with TR. Lags $(1,3,5,7,15-16,19,23$, and 35) values of NR had 
significantly negative association with NR. In ABS estimator, lags (1, 2, 4, 6, 20, 22, 24, 36, and 51-53) values of NR had significantly positive association with NR. Lags $(3,5,7,8,15,19,23$, and 35$)$ values of NR had significantly negative association with NR. Similarly, NC had significantly positive association with NR in USA county-level units. ND had significantly negative association with NR in USA state-level units and county-level units.

The dynamic panel regression results between NC, ND, and NR in the world are presented in Table 5. In AB estimator, lags (1-3, 5-8, 13, 21, 23, 28, 29, 37, 44, 50, 51, $53,57,58,60,66$, and 67 ) values of NR had significantly positive association with NR. Lags $(4,9-11,14,17,18,20,24,25,27,32-34,40,43,45,46,48,54,55,61,64$, and 69) values of NR had significantly negative association with NR. In ABS estimator, lags $(1-3,5-8,16,23,28,31,37,41,44,50,53,57,58,66$, and 67$)$ values of NR had significantly positive association with using NR. Lags (4, 9-11, 13, 17, $20-22,26,33,34,36,38,40,43,45,46,48,54,61$, and 64) values of NR had significantly negative association with NR. Similarly, NC had significantly positive associations with NR in the world. ND had significantly positive association with NR in $\mathrm{ABB}$ estimator in the world.

[Table 5]

\section{Panel event study}

Figure 1 shows that ND increase and then decline steadily over the twenty days 
following the key event. Figure 2 shows that TD decline steadily over the twenty days and increase rapidly following the key event. Figure 3 shows that TD decline steadily over the twenty days and increase rapidly following the key event. Figure 4 shows that TD decline steadily over the twenty days and increase rapidly following the key event. Figure 5 shows that the effect of the key event on NR in USA States is a relatively precisely estimated zero, reflecting both the lack of correlation between NR and key event and the relatively small number of NRs. Figure 6 shows that the effect of the key event on NR in USA Counties is a relatively precisely estimated zero, reflecting both the lack of correlation between NR and key event and the relatively small number of NRs. Figure 7 shows that NR decline steadily over the twenty days and increase rapidly following the key event.

Figure 1. Effect of the key event on ND in China Provinces.

Figure 2. Effect of the key event on TD in China Provinces.

Figure 3. Effect of the key event on NH in China cities.

Figure 4. Effect of the key event on TH in China cities.

Figure 5. Effect of the key event on NR in USA States. 
Figure 6. Effect of the key event on NR in USA Counties

Figure 7. Effect of the key event on NR in The world (outside Antarctica, China, and USA)

\section{Discussion}

This study employed public available daily data in China, USA, and the world (outside Antarctica, China, and USA) and obtained the nexus between NC and ND, the nexus between NC and NR, and the nexus between NC, ND, and NR. Pooled regression showed the association between confirmed and dead cases, and the association between ND and NR, the association between NC and NR. Dynamic regression showed that high orders of lag terms had significant associations with ND, TD, NH, TH, and NR. In panel event study, curve lines showed key events influence $\mathrm{ND}, \mathrm{TD}, \mathrm{NH}$, and $\mathrm{TH}$ significantly, while straight line showed key events had no significant influence on NR.

This study was in agreement with several studies of COVID-19 speed of geographical expansion. Another theoretical study mathematically demonstrated that the relatively high per-capita rate of transmission and the low rate of changes in behavior had caused a large-scale transmission of COVID-19 spatially [23]. Due to transmission between persons, the COVID-19 speed of geographical expansion overwhelmed the increase supply system of public health service in the national regions. This study was in line with another study which revealed that the effect of 
NC on the ND was heterogeneous across Provinces/States in China [24]. But, the finding in this study is not consistent with their results that an increase in NC by $1 \%$ increases ND by $0.10 \%-1.71 \%(95 \% \mathrm{CI})$. This was the same in USA and the world.

This study is in line with several theoretical studies. Traditional public health measures are effective in reducing peak incidence and global deaths of COVID-19 [25]. Therefore, combining emergency response with regular prevention and control measures could compete the tough and long battle against the COVID-19 epidemic.

This study is in line with several empirical studies. For example, an exploratory data analysis with visualizations has been made to understand the number of different cases reported (confirmed, death, and recovered) in different provinces of China and outside of China [26]. Regarding the results in the dynamic regression, this study is congruent with several theoretical studies. In particular, the lags of time series study could be explained by the estimated R0 was between 2.7 and 4.2 [27]. Higher orders of lags indicated that countries of the world should take a series of continuous and austere measures to contain the spread of COVID-19.

The explanations of ineffective policy invention could be over freedom of lifestyle. Facing covid-19 pandemic, a growing number of countries announce at war against further development of contagion. In-house isolation, quarantine, and promoting general awareness about transmission routes are the practical strategies used to tackle the spread of COVID-19 [28]. But, the common persons do not heeded government advice about the importance of individual behavioral attitudes to counter disease propagation. Consequently, the situation rapidly deteriorated with increasing 
number of cases that started to overwhelm health services [29], especially in western countries. The other causes to ineefective prevention and controlling COVID-19 were ill-prepared countries [30], facemask shortage [31], and poor traveller screening [32].

\section{Strengths and weaknesses of the study}

Regarding data sources, this study employed three data. The current study has a large sample size which increased the precision of the study. Additionally, the 60-day period could provide reliable results regarding epidemic control and daily changes in the prevalence of COVID-19 conditions. Regarding statistical methods, this study adopted three advanced panel regression methods. Especially, the event study with difference in difference is used to analyze the role of key events.

There are several limitations. First, several variables included demographics, financial support, and international aids were not taken into account. Statistically, a study in South Korea found that sex, region, and infection reasons affected on both the reported numbers of recovered and deceased cases [33]. Uncontrollable COVID-19 spread in India may be due to inadequately equipped and dedicated health facilities (e.g., space, infection control, waste disposal, safety of healthcare workers, partners to be involved in design and plan) to manage sick patients while protecting healthcare workers and the environment[34]. Second, the spread and transmission of COVID-19 were not considered mathematically and globally. Changes in case definitions affected inferences on the transmission dynamics of COVID-19 allow detection of more cases as knowledge increased in China [35]. 


\section{Conclusions}

Using panel analysis and data collected in China province-level units and city-level units, USA state-level units and county-level units, and The World (outside Antarctica, China, and USA), this study showed China's successful experiences of COVID-19 is worth learning and the pandemic is controllable in the foreseeable future. This study indicated that the other countries should take effective control measures similar to China and therefore keep cautiously optimistic.

\section{LIST OF ABBREVIATIONS}

COVID-19= coronavirus disease 2019

$\mathrm{TC}=$ total confirmed cases

$\mathrm{TD}=$ total dead cases

$\mathrm{ND}=$ newly dead cases

$\mathrm{NC}=$ newly confirmed cases

$\mathrm{NH}=$ newly healed cases

$\mathrm{TH}=$ total healed cases

$\mathrm{NR}=$ newly recovered cases

$\mathrm{AB}$ estimator $=$ Arellano and Bond estimator

ABS estimator=Arellano and Bover/Blundell and Bond system estimator 


\section{Declarations}

\section{Funding}

Not applicable.

Ethics approval and consent to participate

Not applicable.

\section{Consent for publication}

Not applicable.

Availability of data and material

https://dataverse.harvard.edu/dataverse/2019ncov

\section{Competing Interests}

The author declared no potential conflict of interest with respect to the research, authorship and/or publication of this article.

\section{Authors' contributions}

MG designed the study, performed the statistical analysis, and completed the original version.

Corresponding author

Correspondence to Ming Guan.

\section{Acknowledgement}


The author of this paper would like to acknowledge the very helpful comments of two reviewers on the original submission.

\section{Authors' information}

Ming Guan is head of the International Issues Center and Family Issues Center at Xuchang University. He is interested in health care service, health change, and quality of life of migrants and elders in modern China.

\section{Affiliations}

International Issues Center, Xuchang University, Road Bayi, Xuchang, 88, Henan,

China

Ming Guan

Family Issues Center, Xuchang University, Road Bayi, Xuchang, 88, Henan, China Ming Guan

School of Business, Xuchang University, Road Bayi, Xuchang, 88, Henan, China

Ming Guan

\section{Reference}

[1] Gabutti G, d'Anchera E, Sandri F, Savio M, Stefanati A. Coronavirus: Update 
Related to the Current Outbreak of COVID-19. Infect Dis Ther. 2020 Apr 8. doi: 10.1007/s40121-020-00295-5. [Epub ahead of print]

[2] Ford N, Vitoria M, Rangaraj A, Norris SL, Calmy A, Doherty M. Systematic review of the efficacy and safety of antiretroviral drugs against SARS, MERS or COVID-19: initial assessment. J Int AIDS Soc. 2020 Apr;23(4):e25489. doi: 10.1002/jia2.25489.

[3] Wang KW, Gao J, Wang H, Wu XL, Yuan QF, Guo FY, Zhang ZJ, Cheng Y. Epidemiology of 2019 novel coronavirus in Jiangsu Province, China after wartime control measures: A population-level retrospective study. Travel Med Infect Dis. 2020 Apr 5:101654. doi: 10.1016/j.tmaid.2020.101654.

[4] Sanche S, Lin YT, Xu C, Romero-Severson E, Hengartner N, Ke R. High Contagiousness and Rapid Spread of Severe Acute Respiratory Syndrome Coronavirus 2. Emerg Infect Dis. 2020 Apr 7;26(7). doi: 10.3201/eid2607.200282.

[5] Yen MY, Schwartz J, Chen SY, King CC, Yang GY, Hsueh PR. Interrupting COVID-19 transmission by implementing enhanced traffic control bundling: Implications for global prevention and control efforts. J Microbiol Immunol Infect. 2020 Mar 14. pii: S1684-1182(20)30071-2. doi: 10.1016/j.jmii.2020.03.011.

[6] Tian H, Liu Y, Li Y, Wu CH, Chen B, Kraemer MUG, Li B, Cai J, Xu B, Yang Q, Wang B, Yang P, Cui Y, Song Y, Zheng P, Wang Q, Bjornstad ON, Yang R, Grenfell BT, Pybus OG, Dye C. An investigation of transmission control measures during the first 50 days of the COVID-19 epidemic in China. Science. 2020 Mar 31. pii: eabb6105. doi: 10.1126/science.abb6105. [Epub ahead of print] 
[7] Ji T, Chen HL, Xu J, Wu LN, Li JJ, Chen K, Qin G. Lockdown contained the spread of 2019 novel coronavirus disease in Huangshi city, China: Early epidemiological findings. Clin Infect Dis. 2020 Apr 7. pii: ciaa390. doi: 10.1093/cid/ciaa390. [Epub ahead of print]

[8] Amanat F, Krammer F. SARS-CoV-2 Vaccines: Status Report. Immunity. 2020 Apr 14;52(4):583-589. doi: 10.1016/j.immuni.2020.03.007. Epub 2020 Apr 6.

[9] El-Aziz TMA, Stockand JD. Recent progress and challenges in drug development against COVID-19 coronavirus (SARS-CoV-2) - an update on the status. Infect Genet Evol. 2020 Apr 19:104327. doi: 10.1016/j.meegid.2020.104327.

[10] Rahman MS, Peeri NC, Shrestha N, Zaki R, Haque U, Hamid SHA. Defending against the Novel Coronavirus (COVID-19) Outbreak: How Can the Internet of Things (IoT) help to save the World? Health Policy Technol. 2020 Apr 22. doi: 10.1016/j.hlpt.2020.04.005. [Epub ahead of print]

[11] Wells CR, Sah P, Moghadas SM, Pandey A, Shoukat A, Wang Y, Wang Z, Meyers LA, Singer BH, Galvani AP. Impact of international travel and border control measures on the global spread of the novel 2019 coronavirus outbreak. Proc Natl Acad Sci U S A. 2020 Mar 31;117(13):7504-7509. doi: 10.1073/pnas.2002616117. Epub 2020 Mar 13.

[12] Chinazzi M, Davis JT, Ajelli M, Gioannini C, Litvinova M, Merler S, Pastore Y Piontti A, Mu K, Rossi L, Sun K, Viboud C, Xiong X, Yu H, Halloran ME, Longini IM Jr, Vespignani A. The effect of travel restrictions on the spread of the 2019 novel coronavirus (COVID-19) outbreak. Science. 2020 Mar 6. pii: eaba9757. doi: 
10.1126/science.aba9757. [Epub ahead of print]

[13] Leung K, Wu JT, Liu D, Leung GM. First-wave COVID-19 transmissibility and severity in China outside Hubei after control measures, and second-wave scenario planning: a modelling impact assessment. Lancet. 2020 Apr 8. pii: S0140-6736(20)30746-7. doi: 10.1016/S0140-6736(20)30746-7.

[14] Anastassopoulou C, Russo L, Tsakris A1, Siettos C. Data-based analysis, modelling and forecasting of the COVID-19 outbreak. PLoS One. 2020 Mar 31;15(3):e0230405. doi: 10.1371/journal.pone.0230405. eCollection 2020.

[15] Wu JT, Leung K, Leung GM. Nowcasting and forecasting the potential domestic and international spread of the 2019-nCoV outbreak originating in Wuhan, China: a modelling study. Lancet. 2020 Feb 29;395(10225):689-697. doi: 10.1016/S0140-6736(20)30260-9. Epub 2020 Jan 31.

[16] Mengyuan Li, Zhilan Zhang, Shanmei Jiang, Qian Liu, Canping Chen, Yue Zhang, Xiaosheng Wang. Predicting the epidemic trend of COVID-19 in China and across the world using the machine learning approach. medRxiv 2020.03.18.20038117; doi: https://doi.org/10.1101/2020.03.18.20038117

[17] Sanders JM, Monogue ML, Jodlowski TZ, Cutrell JB. Pharmacologic Treatments for Coronavirus Disease 2019 (COVID-19): A Review. JAMA. 2020 Apr 13. doi: 10.1001/jama.2020.6019. [Epub ahead of print]

[18] China Data Lab, 2020, "Policies and Regulations", https://doi.org/10.7910/DVN/OAM2JK, Harvard Dataverse, V9, UNF:6:JqS0gAZjLDn7OqaXP/ZvNw== [fileUNF] 
[19] China Data Lab, 2020, "China COVID-19 Daily Cases with Basemap", https://doi.org/10.7910/DVN/MR5IJN, Harvard Dataverse, V18, UNF:6:6UaA0wJ4LY1Cv2AJJQkXRQ== [fileUNF]

[20] China Data Lab, 2020, "US COVID-19 Daily Cases with Basemap", https://doi.org/10.7910/DVN/HIDLTK, Harvard Dataverse, V17, UNF:6:EoMk3PaaWyd/Fe78Vw8gQw== [fileUNF]

[21] China Data Lab, 2020, "World COVID-19 Daily Cases with Basemap", https://doi.org/10.7910/DVN/L20LOT, Harvard Dataverse, V13, UNF:6:VD511Y14oh70r+RohASKnQ== [fileUNF]

[22] Damian Clarke \& Kathya Tapia Schythe, 2020. "EVENTDD: Stata module to panel event study models and generate event study plots," Statistical Software Components S458737, Boston College Department of Economics.

[23] Kim S, Seo YB, Jung E. Prediction of COVID-19 transmission dynamics using a mathematical model considering behavior changes. Epidemiol Health. 2020 Apr 13:e2020026. doi: 10.4178/epih.e2020026. [Epub ahead of print]

[24] Sarkodie SA, Owusu PA. Investigating the cases of novel coronavirus disease (COVID-19) in China using dynamic statistical techniques. Heliyon. 2020 Apr;6(4):e03747. doi: 10.1016/j.heliyon.2020.e03747. Epub 2020 Apr 4.

[25] Wilder-Smith A, Chiew CJ, Lee VJ. Can we contain the COVID-19 outbreak with the same measures as for SARS? Lancet Infect Dis. 2020 Mar 5. pii: S1473-3099(20)30129-8. doi: 10.1016/S1473-3099(20)30129-8. [Epub ahead of print] Review. 
[26]Dey SK, Rahman MM, Siddiqi UR, Howlader A. Analyzing the epidemiological outbreak of COVID-19: A visual exploratory data analysis approach. J Med Virol. 2020 Mar 3. doi: 10.1002/jmv.25743. [Epub ahead of print]

[27] Zhu Y, Chen YQ. On a Statistical Transmission Model in Analysis of the Early Phase of COVID-19 Outbreak. Stat Biosci. 2020 Apr 2:1-17. doi: 10.1007/s12561-020-09277-0. [Epub ahead of print]

[28] Rahimi F, Talebi Bezmin Abadi A. Practical Strategies Against the Novel Coronavirus and COVID-19-the Imminent Global Threat. Arch Med Res. 2020 Mar 27. pii: S0188-4409(20)30287-3. doi: 10.1016/j.arcmed.2020.03.005. [Epub ahead of print]

[29] Ghanchi A. Adaptation of the National Plan for the Prevention and Fight Against Pandemic Influenza to the 2020 COVID-19 Epidemic in France. Disaster Med Public Health Prep. 2020 Apr 7:1-3. doi: 10.1017/dmp.2020.82. [Epub ahead of print] [30] Gilbert M, Pullano G, Pinotti F, Valdano E, Poletto C, Boëlle PY, D'Ortenzio E, Yazdanpanah Y, Eholie SP, Altmann M, Gutierrez B, Kraemer MUG, Colizza V. Preparedness and vulnerability of African countries against importations of COVID-19: a modelling study. Lancet. 2020 Mar 14;395(10227):871-877. doi: 10.1016/S0140-6736(20)30411-6. Epub 2020 Feb 20.

[31] Wu HL, Huang J, Zhang CJP, He Z, Ming WK. Facemask shortage and the novel coronavirus disease (COVID-19) outbreak: Reflections on public health measures. EClinicalMedicine. 2020 Apr 3:100329. doi: 10.1016/j.eclinm.2020.100329. [Epub ahead of print] 
[32] Katelyn Gostic, Ana Cr Gomez, Riley O Mummah, Adam J Kucharski, James O Lloyd-Smith. Estimated Effectiveness of Symptom and Risk Screening to Prevent the Spread of COVID-19. Elife. 2020 Feb 24;9:e55570. doi: 10.7554/eLife.55570.

[33] Al-Rousan N, Al-Najjar H. Data Analysis of Coronavirus CoVID-19 Epidemic in South Korea Based on Recovered and Death Cases. J Med Virol. 2020 Apr 8. doi: 10.1002/jmv.25850. [Epub ahead of print]

[34] Agarwal A, Nagi N, Chatterjee P, Sarkar S, Mourya D, Sahay RR, Bhatia R. Guidance for building a dedicated health facility to contain the spread of the 2019 novel coronavirus outbreak. Indian J Med Res. 2020 Mar 16. doi: 10.4103/ijmr.IJMR_518_20. [Epub ahead of print]

[35] Tsang TK, Wu P, Lin Y, Lau EHY, Leung GM, Cowling BJ. Effect of changing case definitions for COVID-19 on the epidemic curve and transmission parameters in mainland China: a modelling study. Lancet Public Health. 2020 Apr 21. pii: S2468-2667(20)30089-X. doi: 10.1016/S2468-2667(20)30089-X. [Epub ahead of print] 
Figures

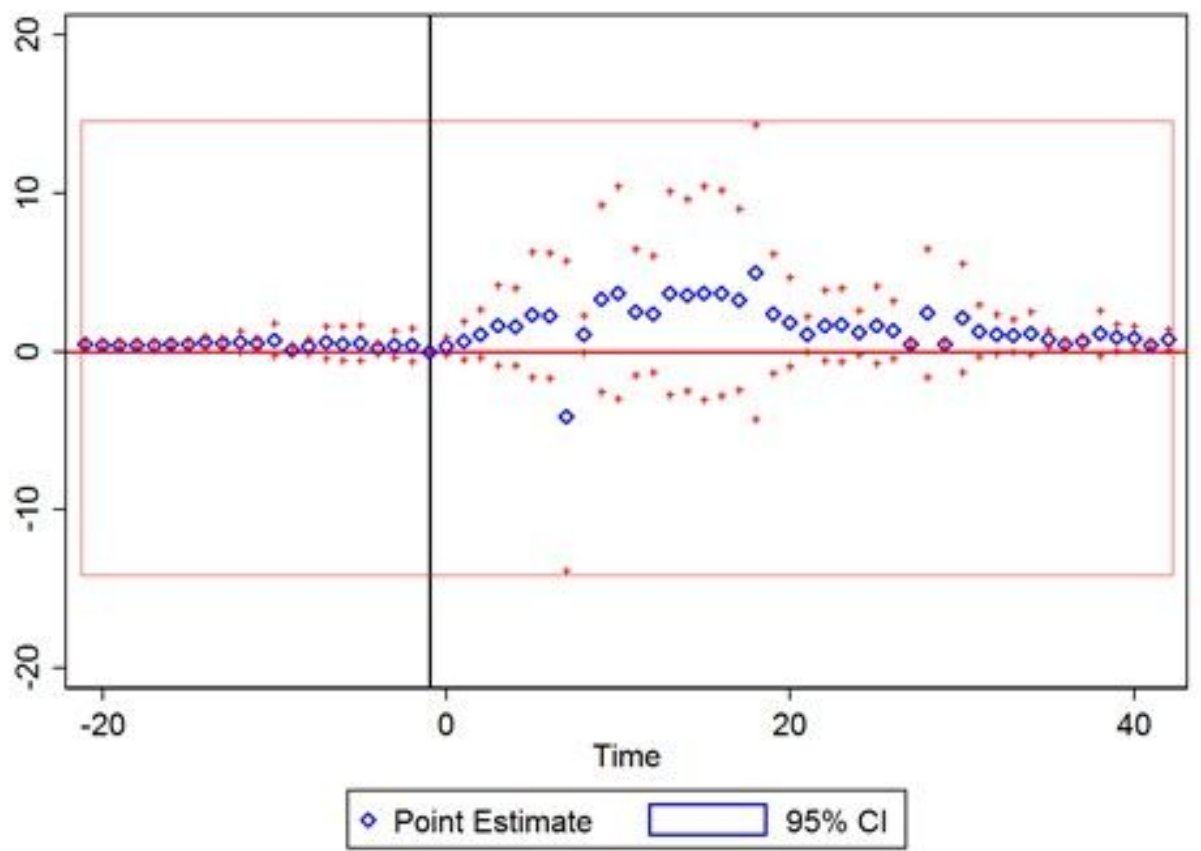

Figure 1

Effect of the key event on ND in China Provinces. 


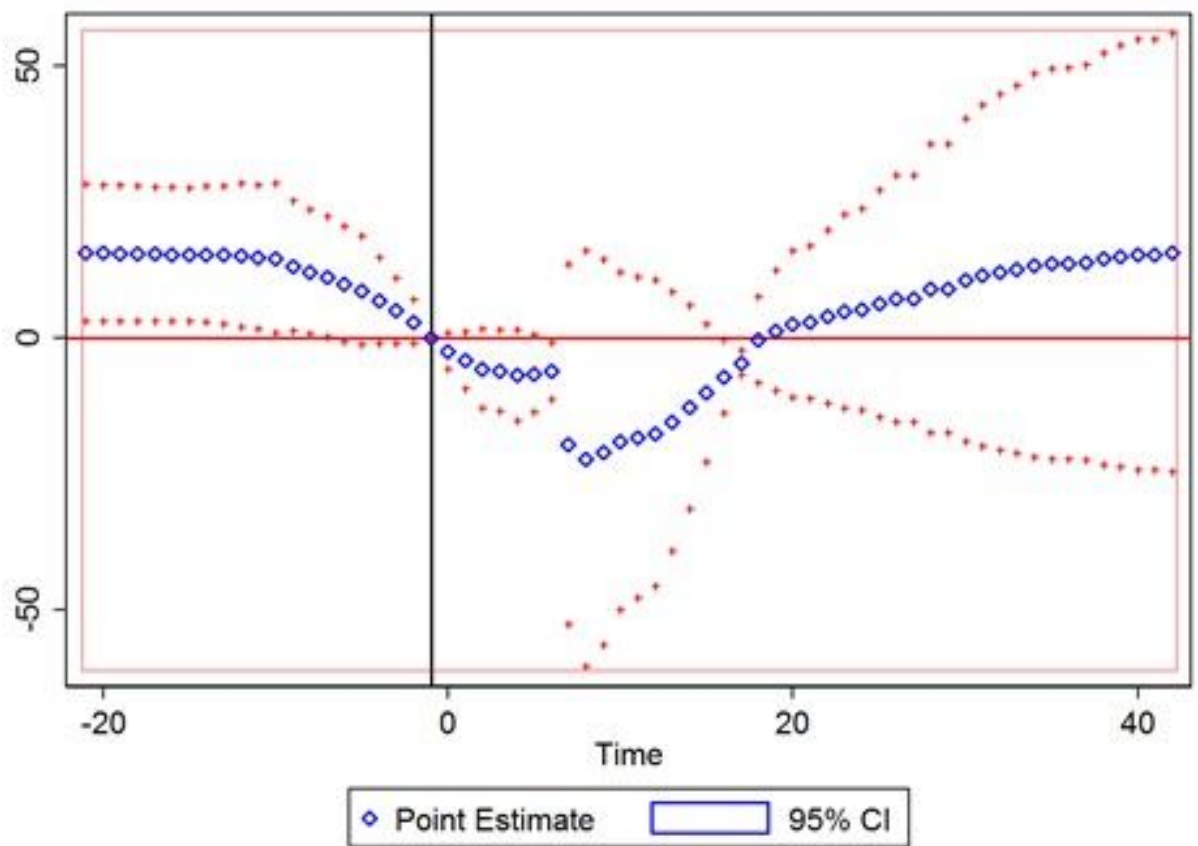

Figure 2

Effect of the key event on TD in China Provinces. 


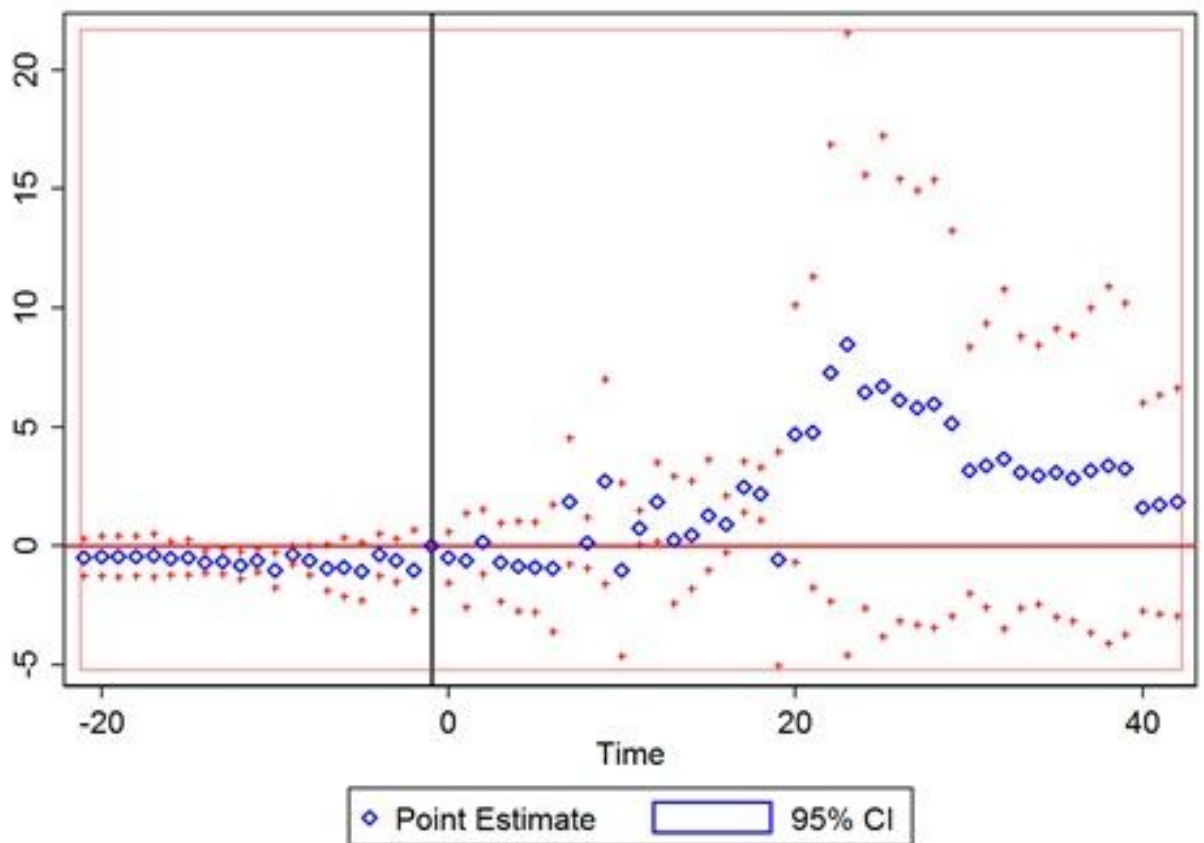

Figure 3

Effect of the key event on $\mathrm{NH}$ in China cities. 


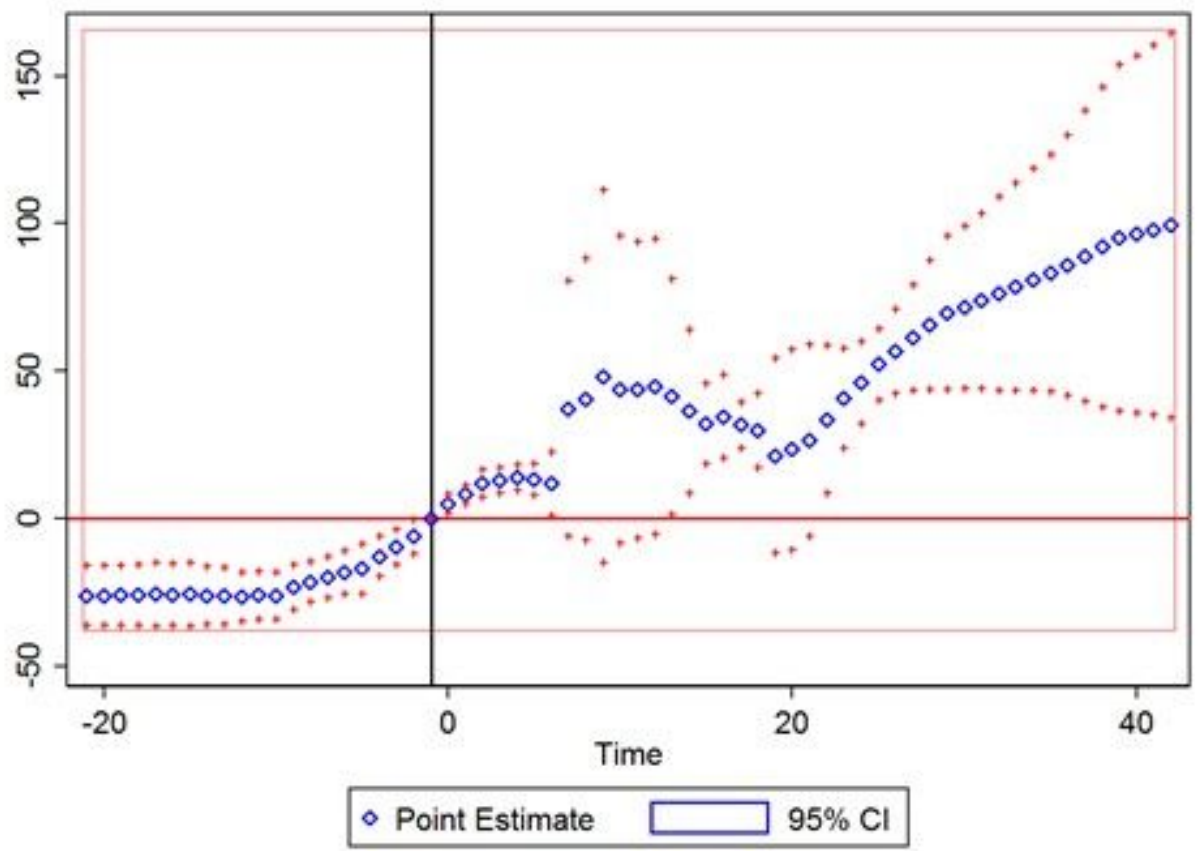

Figure 4

Effect of the key event on TH in China cities. 


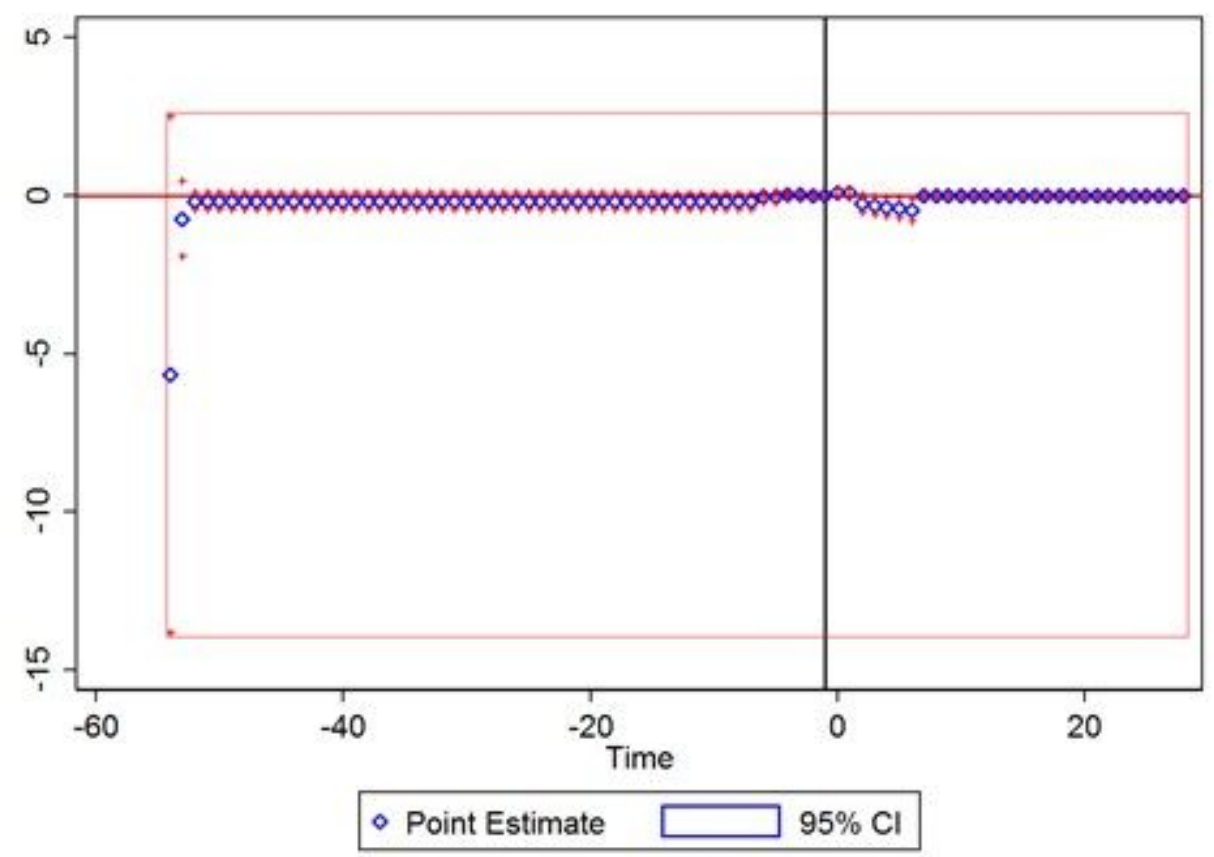

Figure 5

Effect of the key event on NR in USA States. 


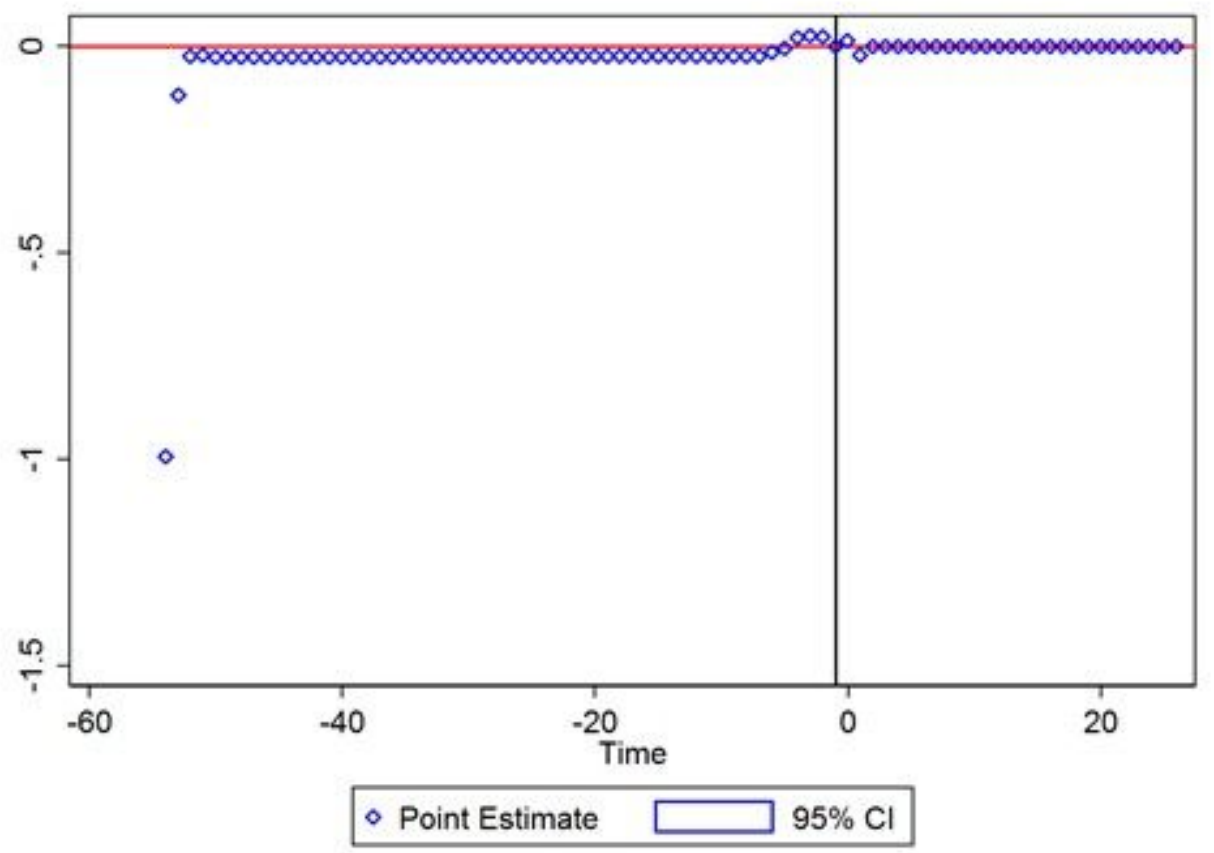

Figure 6

Effect of the key event on NR in USA Counties 


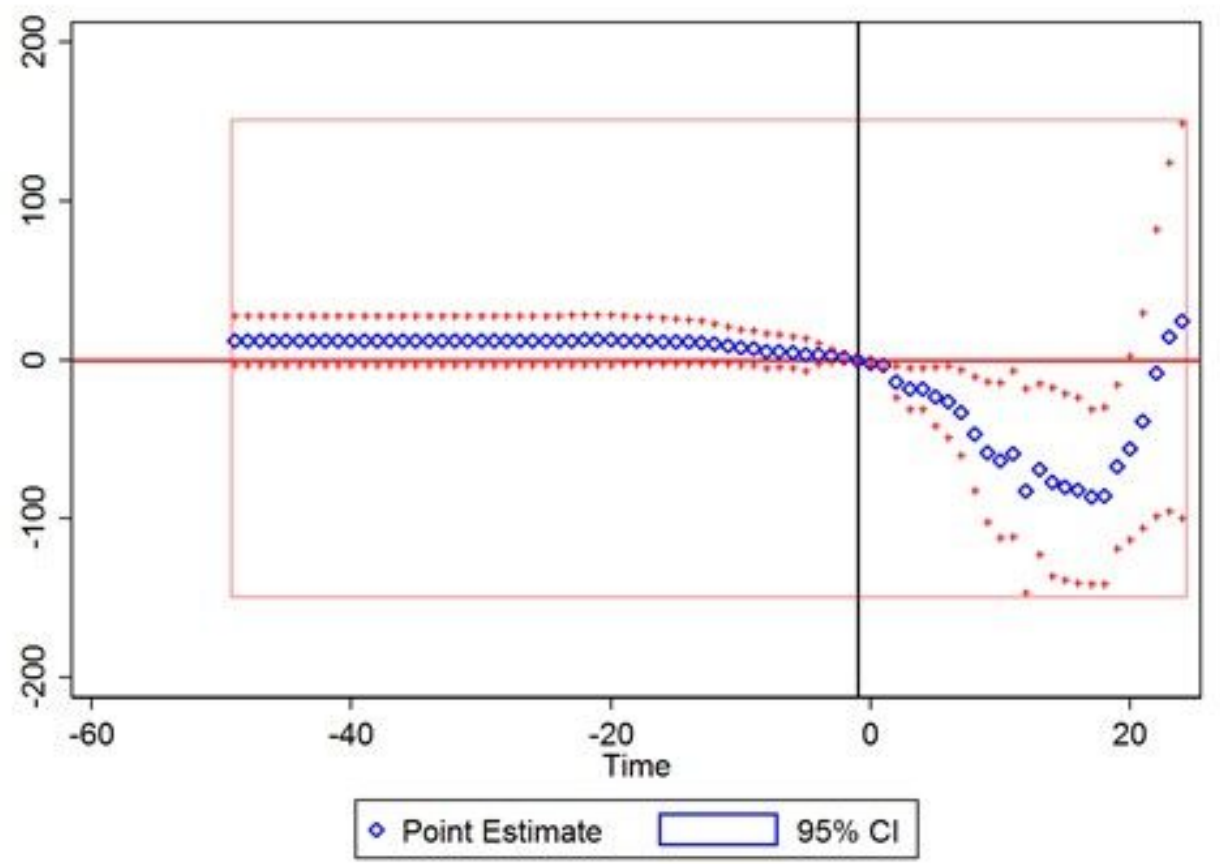

Figure 7

Effect of the key event on NR in The world (outside Antarctica, China, and USA) 